\title{
Cost-effectiveness of pneumococcal conjugate vaccination in Georgia
}

Authors: Komakhidze $\mathrm{T}^{1}$, Hoestlandt $\mathrm{C}^{2}$, Dolakidze $\mathrm{T}^{1}$, Shakhnazarova $\mathrm{M}^{1}$, Chlikadze $\mathrm{R}^{1}$, Kopaleishvili $\mathrm{N}^{3}$, Goginashvili $\mathrm{K}^{4}$, Kherkheulidze $\mathrm{M}^{4}$, Clark $\mathrm{A}^{5}$, Blau $\mathrm{J}^{2}$

\author{
Affiliations \\ ${ }^{1}$ Georgia National Centre for Disease Control and Public Health (NCDC) \\ ${ }^{2}$ Agence de Médecine Préventive (AMP) \\ ${ }^{3}$ National Statistics Office of Georgia (Geostat) \\ ${ }^{4}$ Georgia Ministry of Labor, Health and social affairs $(\mathrm{MoH})$ \\ ${ }^{5}$ London School of Hygene and Tropical Medicine (LSHTM) \\ Corresponding author: Komakhidze T.
}

Email: t.komakhidze@ncdc.ge

\begin{abstract}
Objective: Financial support from the Global Alliance for Vaccines and Immunization (GAVI) to introduce the 10-valent pneumococcal conjugate vaccine (PCV10) into the routine childhood immunization schedule in Georgia is ending in 2015. As a result, the Interagency Coordination Committee (ICC) decided to carry out a cost-effectiveness analysis to gather additional evidence to advocate for an appropriate evidence-based decision after GAVI support is over. The study also aimed to strengthen national capacity to conduct costeffectiveness studies, and to introduce economic evaluations into Georgia's decision-making process.
\end{abstract}


Methodology: A multidisciplinary team of national experts led by a member of the ICC carried out the analysis that compared two scenarios: introducing PCV10 vs. no vaccination. The TRIVAC model was used to evaluate 10 cohorts of children over the period 2014-2033. National data was used to inform demographics, disease burden, vaccine coverage, health service utilization, and costs. Evidence from clinical trials and the scientific literature was used to estimate the impact of the vaccine. A $3+0$ schedule and a vaccine price increasing to US\$ 3.50 per dose was assumed for the base-case scenario. Alternative univariate and multivariate scenarios were evaluated.

Results: Over the 10-year period, PCV10 was estimated to prevent 7,170 (8,288 undiscounted) outpatient visits due to all-cause acute otitis media, 5,325 (6,154 undiscounted) admissions due to all-cause pneumonia, 87 (100 undiscounted) admissions due to pneumococcal meningitis, and 508 (588 undiscounted) admissions due to pneumococcal nonpneumonia and non-meningitis (NPNM). In addition, the vaccine was estimated to prevent 41 (48 undiscounted) deaths. This is equivalent to approximately 5 deaths and 700 admissions prevented each year in Georgia. Over the 10-year period, PCV10 would cost the government approximately US\$ 4.4 million ( $\$ 440,000$ per year). However, about half of this would be offset by the treatment costs prevented. The discounted cost-effectiveness ratio was estimated to be US\$1,599 per DALY averted with scenarios ranging from US\$ 286 to US\$ 7,787. Discussion: This study led to better multi-sectoral collaboration and improved national capacity to perform economic evaluations. Routine infant vaccination against S. Pneumoniae would be highly cost-effective in Georgia. The decision to introduce PCV10 was already made some time before the study was initiated but it provided important economic evidence in support of that decision. There are several uncertainties around many of the parameters used, but a multivariate scenario analysis with several conservative assumptions (including no 
herd effect in older individuals) shows that this recommendation is robust. This study supports the decision to introduce PCV10 in Georgia.

Keywords: Georgia, cost-effectiveness analysis, Pneumococcal conjugate vaccine, decisionmaking process, evidence-based medicine

\section{Introduction} independence from the Soviet Union in 1991. However, over the past decade, the socio-

4 economic situation has improved due to substantial economic and social reforms. While

5 economic growth was adversely affected by the August 2008 conflict, growth rebounded again in 2010 and the World Bank now classifies Georgia as a lower middle-income country. Similarly, over the last 10 years (2002-2012) the infant mortality rate was cut by nearly half, dropping from 20.1 to 10.8 deaths per 1,000 live births, according to national health statistics, and from 23.6 to 12.6 deaths per 1,000 live births, according to vital statistics.

Among the main causes of infant deaths are diseases of the respiratory system, certain conditions originating in the perinatal period, infectious and parasitic diseases, congenital malformations, and external causes such as accidents, injuries, and poisoning. The highest proportion of child morbidity is related to respiratory system diseases. Two efficacious and safe pneumococcal conjugate vaccines (PCV) are currently available to help reduce mortality and morbidity due to pneumonia, meningitis, and other invasive diseases caused by Streptococcus pneumoniae (Spn). Both vaccines can currently be procured with the financial 17 support of the Global Alliance for Vaccines and Immunization (GAVI), but the support ends December 2015 and the government will then bear the total cost of the program. Georgia had 19 planned to introduce the 10-valent pneumococcal conjugate vaccine (PCV10) in 2013 but due 
to healthcare system reorganization and the late introduction of a rotavirus vaccination (RV),

21 a decision was made to postpone introducing PCV until 2014. Therefore, in consultation with the World Health Organization (WHO) Regional Office for Europe, the Interagency

Coordination Committee (ICC), which is the national advisory committee on immunization, decided to conduct a cost-effectiveness analysis (CEA) on the introduction of PCV10 with support from the ProVac International Working Group (IWG). The aim of the study was to inform the government about the economic and epidemiological impact of introducing PCV10. A multidisciplinary team of national experts from several organizations (including and led by a member of the ICC), was established and met regularly to collect and review the best available national data. The team conducted the CEA to evaluate whether or not introduction of PCV10 would be cost-effective compared to no introduction, and through this process, to decide whether or not to advocate for an appropriate evidence-based decision after GAVI support has ended.

\section{Methods}

The ProVac Initiative was begun in the Americas by the Pan American Health Organization (PAHO) and during a two-year pilot phase, it was expanded to other regions through the ProVac IWG. [1]. Georgia was supported by the Agence de Médecine Préventive (AMP), an implementing partner of the ProVac IWG, in collaboration with WHO Europe.

The TRIVAC decision support model, version 2.0, was used. This is an internationally recognized transparent Excel®-based model, described in detail elsewhere [2]. The

40 parameters of the model required collection of data that included demography, burden of 41 disease, health services utilization and costs, vaccine coverage, vaccine efficacy, and vaccination program costs. 

universal introduction of the PCV10 vaccination into the Georgian National Immunization Program and (2) no PCV vaccination. Ten consecutive births cohorts were modeled over the period 2014- 2023. Following WHO recommendations, a 3\% discount rate was used for both costs and health outcomes [3]. We assumed that PCV10 would be administered in three primary doses without a booster dose $(3 p+0)$. Realistic estimates of vaccination coverage and timeliness were based on estimates available for DTP vaccination. Four clinical outcomes related to Streptococcus pneumoniae were included: (1) outpatient visits due to all-cause acute otitis media (AOM), (2) inpatient admissions due to all-cause pneumonia, (3) inpatient admissions due to pneumococcal meningitis, and (4) inpatient admissions due to pneumococcal non-pneumonia non-meningitis (NPNM) invasive disease. It was the opinion of expert members of the team, in conjunction with a consensus among public health experts and pediatricians, that all cases of severe pneumonia, pneumococcal meningitis and pneumococcal NPNM would be admitted to a hospital in Georgia; as a result, it was practical and reasonable to focus on admissions rather than all cases in the community. A government perspective was taken, so no household costs were considered. In Georgia, the majority of

60 providers are private and costs of treatment are partly or fully covered by the government. Three types of providers were taken into account: primary care providers (private individual entrepreneurs, who receive salaries from government), secondary care (polyclinics), and tertiary care (hospitals). All three providers handle outpatient visits for AOM, but only hospitals (tertiary care) handle inpatient admissions for invasive pneumococcal disease.

\subsection{Demography}


on the projection of the United Nations Population Division [UNPOP]) was applied to this figure to estimate the number of births in future years. To take into consideration the uncertainty around the assumption of a declining population, a second scenario with no yearover-year trend was also evaluated. Estimates on mortality rates for infants and children younger than 5 years of age were provided by the National Centre for Disease Control and Public Health (NCDC). In 2012, 14 of every 1,000 live births died before the age of 1; 16 of every 1,000 live births died before the age of 5. The life expectancy for those born in 2012 was assumed to be 75 . This was used to estimate the number of life-years lost and disabilityadjusted life-years (DALY) due to mortality related to Streptococcus pneumoniae. The UNPOP projections for Georgia were applied to these estimates to generate a projection for the period 2014-2023 [3].

\subsection{Burden of disease}

Data concerning AOM and pneumonia were based on national statistical reports published in the statistical yearbook 2012. Two types of reports were available to estimate AOM incidence; therefore, two estimates of incidences were calculated. Both report types gather data from health facilities based on the International Classification of Diseases (ICD) $10^{\text {th }}$ revision codes. However, one is from the Tbilisi-level case-based reporting system and the other one is from the national reporting system of notifiable diseases. These two reporting systems were used to estimate a Tbilisi annual incidence of 1,695 cases of AOM per 100,000 under 5 years old and a national annual incidence of 3,073 per 100,000 under 5 years. The more conservative Tbilisi incidence was used in the base-case analysis; the national incidence was used in the scenario analysis. The national-level reporting was sufficient to estimate the incidence of pneumonia admissions, 1,603 per 100,000 under 5 years old. For pneumococcal meningitis and pneumococcal NPNM, no national data were available; instead, WHO 2008 
estimates for Georgia were used [4], with annual incidences, respectively, of 10 and 62 per 100,000 under 5 years.

Data on the number of pneumonia deaths was based on the national reporting system. A case fatality ratio (CFR) was calculated by dividing the number of pneumonia deaths by the estimated number of pneumonia admissions. The CFR of $25 \%$ for pneumococcal meningitis was based on estimates from sentinel surveillance for bacterial meningitis. The CFR for pneumococcal NPNM was based on the WHO estimate for Georgia. No national data were available on the proportion of children with long-term sequelae following meningitis; instead, global estimates from Edmond et al. were used [5]. In order to estimate DALYs, each syndrome was assigned a disability weight provided by the WHO global burden of disease (GBD) [4]. The age distribution of pneumococcal disease was based on estimates for Europe by Sanderson et al. [14] and mean duration of illness was estimated after consulting national experts.

\subsection{Health services cost related to Streptococcus pneumoniae (Spn)}

Only government expenditures were taken into consideration. Based on data from Ministry of Health $(\mathrm{MoH})$ state health programs, the weighted average cost of a visit for AOM was equivalent to US\$ 11.84 . This sum was calculated from two costs: professional services and lab exams. In addition, the costs of AOM-related visits were inflated to reflect the fact that some of the AOM cases are indeed hospitalized and incur substantial cost. The visit cost was assumed to be equal for all three types of providers. According to the $\mathrm{MoH}$, the cost of one hospital admission for pneumonia was estimated at US\$ 301.18, for pneumococcal meningitis at US\$ 494.12, and for pneumococcal NPNM at US\$ 800.00.

14 Sequelae costs borne by the government were not considered due to lack of data. 
Coverage of PCV1 over the 2014-2023 period was assumed to be $67 \%, 75 \%, 87 \%$, $90 \%, 94 \%, 96 \%$, and $98 \%$ thereafter. This was based on data on DTP immunization coverage for 2013 obtained from the national immunization reporting system and provided by the NCDC immunization department. Rotavirus vaccine coverage was used to inform the estimates of PCV coverage in the first few years, as this vaccine was introduced relatively recently. Estimated coverage of DTP doses 1, 2, and 3 was used as a proxy for the expected drop-out rate between PCV doses 1, 2, and 3. The timeliness of PCV doses 1, 2, and 3 was based on estimates for the European region by Clark and Sanderson [14]. Two studies were considered for PCV10 vaccine efficacy against all-cause AOM. One was from Czech and Slovak Republic [6]; the second was a study nested [7] within a recent clinical trial from Finland [8]. The evidence from the study in the Czech and Slovak Republic, $33.6 \%(20.8 \%$; 44.3\%), was assumed for the base case. For efficacy against all-cause pneumonia, $26.2 \%$ efficacy was assumed based on a meta-analysis of the fraction of radiological pneumonia (proxy for pneumonia admissions) prevented in clinical trials in

130 California, the Philippines, and South Africa [4]. The estimate from the Gambian trial was much higher than in the other three trials and since it was not considered relevant to the

132 Georgian context, it was excluded. For pneumococcal meningitis and NPNM, we used 81\% vaccine efficacy based on the Cochrane review [9] and we considered the uncertainty around

134 these estimates in the scenario analysis.

There is a good deal of uncertainty around assumptions for herd effects, serotype

136 replacement, waning vaccine-induced protection, and relative coverage (i.e., the extent to 137 which high-risk children are covered by the vaccine). We therefore varied these assumptions 138 in scenario analyses to assess their influence on the cost-effectiveness result. For the base 139 case, we assumed 95\% relative coverage by dividing DTP2 coverage in the poorest quintile 
140 by DTP2 coverage in the entire birth cohort. Overall program coverage was then multiplied

141 by $95 \%$ to provide a more realistic estimate of coverage for children at the highest risk of

142 mortality from invasive pneumococcal disease. The 95\% value was based on estimates from

143 Demographic and Health Surveys (DHS) in other middle-income countries [15]. We assumed

144 that the direct effectiveness in children younger than 5 could be multiplied by $120 \%$ to reflect

145 the observed herd effect in this age group with PCV7 in the USA [10].We did not consider

146 herd effects in older age groups. Waning vaccine-induced protection and type replacement

147 were assumed to be 5\% and 5.5\% per year, respectively, but these were both varied in

148 scenario analyses.

\subsection{Vaccination program cost}

To estimate the price of the vaccine and injection supplies, we used NCDC data and

151 the country GAVI proposal for introducing PCV. In the base-case scenario we used a per-

152 dose vaccine price of US\$ 3.50, which was then adjusted for the initial years because national

153 co-financing is progressive (see Table 4). The additional freight cost was taken at $5 \%$ of the

154 vaccine price. Based on the NCDC data, the wastage rate for the two-dose vaccine

155 presentation was assumed to be $10 \%$. Prices for safety boxes and administering syringes were 156 also considered.

157 The incremental system cost per dose for introducing PCV vaccine was estimated

158 based on the country comprehensive multi-year plan (cMYP) 2010-2015. The per-dose

159 spending was estimated to be US\$ 0.63 . For the first year of introduction, the team calculated

160 the difference between expected costs in 2012 and in 2013; the only difference between these

161 two years was the expected introduction of PCV10. This cost was divided by the number of

162 doses (with a $3+0$ schedule) expected in the first year of introduction. Using this data, we 
163 estimated the operational cost of the launch to be US\$ 1.06 in the first year and US\$ 0.60 in

164 subsequent years.

\subsection{Scenario analysis}

A scenario analysis was performed to evaluate the uncertainty of results due to the

167 shortage of reliable national data. The scenarios tested were: (1) high and low burden of 168 disease (i.e., incidence and deaths), (2) no change in the number of births over time, (3) high

169 and low vaccine efficacy, (4) waning vaccine-induced protection, (5) high and low health

170 services costs (i.e., outpatients and inpatients), (6) no discount rate, (7) worst case

171 (unfavorable for introduction), (8) best case (favorable for introduction), and (9) high and low

172 coverage. We also considered a scenario in which no adjustment was made for herd effects or

173 type replacement.

\section{3. Results}

\subsection{Without vaccination}

With no introduction of PCV10 over the period 2014-2023, we estimate 34,444

177 (39,838 undiscounted) outpatient visits due to all-cause AOM, 32,578 (37,680 undiscounted)

178 admissions due to all-cause pneumonia, 213 (247 undiscounted) admissions due to

179 pneumococcal meningitis, and 1,250 (1,446 undiscounted) admissions due to pneumococcal

180 NPNM. In addition, there would be 4,744 discounted DALYs and US\$ 11.33 million

181 discounted health service costs borne by the government.

184 summarized in Table 6. In brief, over the period 2014-2023, PCV10 is estimated to prevent 

admissions for all-cause pneumonia, 87 (100 undiscounted) admissions for pneumococcal meningitis and 508 (588 undiscounted) admissions for pneumococcal NPNM. In addition, the vaccine is estimated to prevent 41 (48 undiscounted) deaths. This is equivalent to preventing approximately 5 deaths and 700 admissions each year in Georgia.

Over the 10-year period, PCV10 would cost the government approximately US\$ 4.4

191 million (US\$ 440,000 per year). However, about half of this would be offset by averted treatment costs of approximately US\$2.14 million. (Of that figure, US\$ 1.6 million are averted treatment costs for pneumonia.) The net cost borne by government would be equal to US\$2.30 million. This amount is derived from the total cost of vaccination minus the savings

195 from averted treatment (Table 8). The incremental cost-effectiveness ratio (i.e., the cost per

196 DALY averted) from governmental perspective is equal to US\$ 1,599 per DALY averted.

197 According to the WHO recommendation of using the gross domestic product (GDP) per 198 capita as thresholds to interpret this ratio, the introduction of PCV in Georgia is highly cost199 effective, which means that taking into account the end of GAVI's support, the intervention is 200 still a valuable investment for the government [12].

All results of the scenario analysis are shown in Figure 1. Almost all are below the GDP per capita threshold (i.e., highly cost-effective). Even the worst-case scenario, which gathers unfavorable figures of several parameters, still results in a cost-effective intervention.

204 Therefore, from the governmental perspective, the results of this analysis are robust.

\section{Discussion}

This study assessed the potential impact of introducing the vaccine in terms of health

207 benefits, incremental costs to the immunization program, health system costs averted, and 208 cost-effectiveness of the introduction of the pneumococcal vaccine. These criteria are all 
relevant to the decision-making process in Georgia. This analysis shows that introduction of

210 PCV10 is likely to be highly cost-effective in Georgia. It also provides evidence about the

211 clinical and economical burden of the Streptococcus pneumoniae in Georgia, and about the

212 benefit of introducing PCV10 to the national immunization program. The introduction of

213 PCV10 is estimated to cost approximately US\$ 4-5 million over a decade, but about half this

214 cost would be offset by savings from reduced hospital admissions and outpatient visits.

This cost-effectiveness analysis is the first country-led economic evaluation of a vaccine in Georgia. Utilization of a transparent model to perform the study made it possible to obtain useful evidence and multiple relevant results. The methodology used to collect data included several experts from different fields and was an effective way to produce a snapshot

219 of the current economic and epidemiological burden associated with pneumococcal disease in Georgia.

However, the study faced some limitations. (1) Data on community pneumonia were not available, so no outpatient visits were assumed for pneumonia; this resulted in underestimating the burden of disease. (2) Only expert opinion was available for estimates of 224 parameters such as duration of illness or health service utilization; because no local data were available for sequelae and associated costs, the benefits of vaccination were underestimated. (3) The societal perspective was not explored due to lack of available data and also due to the recent change in the health financing system. (4) Herd immunity effect among people older than 5 years old, especially elderly people, was not modeled although the herd effect is likely

229 to have a significant impact on the cost-effectiveness and would make the result even more 230 favorable. (5) Although global estimates used for incidence of pneumococcal meningitis and 231 NPNM are reasonable placeholders, a good quality national source would be more 232 appropriate. 
234 scenario analyses and the fact that the vaccine remained cost-effective despite very

235 conservative assumptions (e.g., no herd effect in older individuals) suggests that the

236 conclusion is robust. In addition, it should also be mentioned that AOM inclusion

237 would improve CE ratio further.

238 Other benefits of the national study included the identification of important gaps in 239 national data, such as health service utilization and costs, and the increased capacity of the 240 national team to conduct economic evaluations in the future.

\section{5. Conclusion}

The decision to introduce PCV10 was already made some time before the study was

243 initiated but this study provides important economic evidence in support of that decision. Not

244 only is it highly cost-effective, it is also likely to prevent approximately 5 child deaths and a

245 substantial number of hospital admissions annually. This analysis also created the opportunity

246 to identify some gaps in national data, especially on health care utilization, for which we

247 relied mainly on expert opinion. It is important to work on the collection of these data. This

248 analysis was a good capacity building process and led to better understanding of cost-

249 effectiveness, better multi-sectorial collaboration and an improved national capacity to

250 perform the economic evaluations. In conclusion, it would be interesting to extend this type of

251 evaluation to other health interventions in order to compare their cost-effectiveness and

252 provide a new source of information for decision-makers.

\section{Conflict of interest}


255 supported by the ProVac IWG, which is funded entirely by a grant from the Bill \& Melinda 256 Gates Foundation.

\section{Acknowledgements}

259 Pilishvili from US CDC, and Dr. Viktor Galayda for fruitful collaboration and their

260 contribution to this study. We would also like to thank Giorgi Kurtsikashvili from WHO CO,

261 and Chris Duggar and Julia Morgan and Add Maes from US CDC for their kind contributions, 262 as well as the team from the NCDC immunization department. 


\section{References}

1. Jauregui B, Sinha A, Clark AD, Bolanos BM, Resch S, Toscano CM, Matus CR, and Andrus JK. Strengthening the technical capacity at country-level to make informed policy decisions on new vaccine introduction: lessons learned by PAHO's ProVac Initiative, Vaccine, January 2011, Vol. 29, Issue 5, 1099-1106.

2. Clark A, Jauregui B, Griffiths U, Janusz CB, Bolaños-Sierra B, and Hajjeh R et al. TRIVAC decision-support model for evaluating the cost-effectiveness of Haemophilus influenzae type b, pneumococcal and rotavirus vaccination. Vaccine. 2013;31:C19-C29.

3. WHO guide for standardization of economic evaluations of immunization programs, World Health Organization, 2008 (WHO/IVB/08.14).

4. O'Brien KL, Wolfson LJ, Watt JP, Henkle E, Deloria-Knoll M, McCall N, Lee E, Mulholland K, Levine OS, and Cherian T. Burden of disease caused by Streptococcus pneumoniae in children younger than 5 years: global estimates, The Lancet, September 2009, Vol. 374, No 9693, 893-902.

5. Edmond K, Clark A, Korczak VS, Griffiths UK, and Rudan I. Global and regional risk of disabling sequelae from bacterial meningitis: a systematic review and meta- analysis. Lancet Infect Dis. 2010; 10:317-28.

6. Prymula R, Peeters P, Chrobok V, Kriz P, Novakova E, and Kaliskova E et al. Pneumococcal capsular polysaccharides conjugated to protein $\mathrm{D}$ for prevention of acute otitis media caused by both Streptococcus pneumoniae and non-typable Haemophilus influenzae: a randomised double-blind efficacy study, The Lancet, 2006, 367(9512): $740-8$.

7. Vesikari T. Effectiveness of the 10-valent pneumococcal non-typeable Haemophilus influenzae protein D conjugate vaccine against acute otitis media. Abstract presented at: 52nd Annual Interscience Conference on Antimicrobial Agents \& Chemotherapy 
(ICAAC); September 9-12, 2012; San Francisco, Calif.

8. Palmu AA, Jokinen J, Borys D, Nieminen H, Ruokokoski E, Siira L, Puumalainen T, Lommel P, Hezareh M, Moreira M, Schuerman L, and Kilpi TM. Effectiveness of the pneumococcal Haemophilus influenzae protein D conjugate vaccine (PHiD-CV10) against invasive pneumococcal disease: a cluster randomised trial Lancet 2013 Jan $19 ; 381(9862): 214-22$.

9. Lucero MG, Dulalia VE, Nillos LT, Williams G, Parreño RA, and Nohynek H et al. Pneumococcal conjugate vaccines for preventing vaccine-type invasive pneumococcal disease and X-ray defined pneumonia in children less than two years of age. Cochrane Database, 2009, Review.

10. Centers for Disease Control and Prevention. Invasive Pneumococcal Disease in Children 5 Years After Conjugate Vaccine Introduction - Eight States, 1998-2005. MMWR.

11. Miller E, Andrews NJ, Waight PA, Slack MP, and George RC. Herd immunity and serotype replacement four years after seven-valent pneumococcal conjugate vaccination in England and Wales: an observational cohort study. Lancet Infect Dis. 2011; 11:760-

12. Sachs J. Macroeconomics and health: investing in health for economic development. World Health Organization; Geneva: 2001.

13. United Nations Population Division (UNPOP). World Population Prospects: The 2012 revision. 2013 Available at: http://esa.un.org/wpp/.

310 14. World Bank. Data: Neonatal Mortality Rates. 2011. Available at: http://data.worldbank.org/indicator/SH.DYN.NMRT. middle-income countries: an analysis of survey data. Lancet, 2009. 373 (9674): p. 1543- 
9.

315 16. London School of Hygiene and Tropical Medicine and ProVac's Centres of Excellence 316 Network. OLIVES (on-line international vaccine economics and statistics), Available 317 at: http://provac-olives.com/olives/ (Accessed: October, 2013). 
Table 1. Input parameters for estimating disease burden

\begin{tabular}{|c|c|c|c|c|}
\hline \multirow[t]{2}{*}{ Parameter } & \multirow[t]{2}{*}{ Estimate } & \multicolumn{2}{|c|}{ Scenarios } & \multirow[t]{2}{*}{ Source/s } \\
\hline & & Low & High & \\
\hline \multicolumn{5}{|l|}{ Annual incidence per 100,000 aged $1-59 \mathrm{~m}$ : } \\
\hline All-cause acute otitis media & 1,695 & 1,695 & 3,073 & National reporting system \\
\hline All-cause pneumonia admissions & 1,603 & 1,603 & 1,603 & Estimate \\
\hline Pneumococcal meningitis admissions & 10.5 & 5.8 & 17.4 & GBD 2000 \\
\hline Pneumococcal NPNM admissions & 61.5 & 33.8 & 102.1 & GBD 2000 \\
\hline \multicolumn{5}{|l|}{$\%$ case fatality ratios (CFRs) in ages $1-59 \mathrm{~m}:^{a}$} \\
\hline All-cause pneumonia admissions & $0.20 \%$ & $0.16 \%$ & $0.24 \%$ & NCDC Georgia \\
\hline Pneumococcal meningitis admissions & $25.0 \%$ & $12.96 \%$ & $29.87 \%$ & Sentinel surveillance (NCDC Geo) \\
\hline Pneumococcal NPNM admissions & $2.6 \%$ & $2.33 \%$ & $5.14 \%$ & GBD 2000 (adjusted to 2014) \\
\hline \multicolumn{5}{|c|}{$\%$ sequelae in pneumococcal meningitis survivors } \\
\hline$\%$ Major sequelae (single) & $20.2 \%$ & - & - & Edmond 2010 \\
\hline \% Major sequelae (multiple) & $4.5 \%$ & - & - & Edmond 2010 \\
\hline \multicolumn{5}{|l|}{ Disability weight for DALY calculations } \\
\hline All-cause acute otitis media & 0.02 & - & - & WHO GBD 1990 \\
\hline All-cause pneumonia admissions & 0.28 & - & - & WHO GBD 1990 \\
\hline Pneumococcal meningitis admissions & 0.62 & - & - & WHO GBD 1990 \\
\hline Pneumococcal NPNM admissions & 0.28 & - & - & Assumption (Griffiths $\mathrm{PhD}$, page 107) \\
\hline$\%$ Major sequelae (single) & 0.24 & - & - & Assumption (Griffiths $\mathrm{PhD}$, page 107) \\
\hline \% Major sequelae (multiple) & 0.63 & - & - & Assumption (Griffiths $\mathrm{PhD}$, page 107) \\
\hline \multicolumn{5}{|l|}{ Mean duration of illness (in days) } \\
\hline All-cause acute otitis media & 6 & - & - & Expert opinion (2) \\
\hline All-cause pneumonia admissions & 6 & - & - & Expert opinion (2) \\
\hline Pneumococcal meningitis admissions & 10 & - & - & Expert opinion (2) \\
\hline Pneumococcal NPNM admissions & 6 & - & - & Expert opinion (2) \\
\hline \multicolumn{5}{|l|}{ Age distribution of disease cases and deaths } \\
\hline$<3 \mathrm{~m}:$ & $9.4 \%$ & - & - & Sanderson 2013, WHO IVR Schedule Report \\
\hline 3-5m: & $13.0 \%$ & - & - & Sanderson 2013, WHO IVR Schedule Report \\
\hline 6-8m: & $13.3 \%$ & - & - & Sanderson 2013, WHO IVR Schedule Report \\
\hline 9-11m: & $12.2 \%$ & - & - & Sanderson 2013, WHO IVR Schedule Report \\
\hline 12-23m: & $32.5 \%$ & - & - & Sanderson 2013, WHO IVR Schedule Report \\
\hline 24-35m: & $13.3 \%$ & - & - & Sanderson 2013, WHO IVR Schedule Report \\
\hline 36-47m: & $4.7 \%$ & - & - & Sanderson 2013, WHO IVR Schedule Report \\
\hline 48-59m: & $1.6 \%$ & - & - & Sanderson 2013, WHO IVR Schedule Report \\
\hline
\end{tabular}

${ }^{a}$ In the absence of vaccination, CFRs are assumed to decline in each successive birth cohort in line with the general trend in mortality under 5 years of age. This is done by assuming that the fraction of deaths under age 5 that are caused by the disease remains fixed over time. 
Table 2. Input parameters for estimating health service utilisation and costs (all costs are presented in US \$) 2013

\begin{tabular}{|c|c|c|c|c|}
\hline Parameter & Estimate & \multicolumn{2}{|c|}{ Scenarios } & Source/s \\
\hline \multicolumn{5}{|l|}{ Government cost per outpatient visit ${ }^{a}$} \\
\hline \multicolumn{5}{|l|}{ INPATIENT ADMISSIONS } \\
\hline \multicolumn{5}{|l|}{ Government cost per inpatient admission ${ }^{b}$} \\
\hline All-cause pneumonia admissions & $\$ 301$ & $\$ 241$ & $\$ 361$ & $\mathrm{MoH}$ \\
\hline
\end{tabular}

${ }^{a}$ Government costs per outpatient visit for AOM included professional services and a lab exam. The costs of AOM visits were also inflated to account for some of these cases being hospitalized. Outpatient visits are distributed as follows: $50 \%$ polyclinic $10 \%$ hospital, $40 \%$ private individual entrepreneur (PIE). The cost presented is the weighted average of the provider-specific costs.

${ }^{b}$ Government costs per inpatient admission include the cost per bed-day multiplied by the expected length of stay, the cost of disease-specific drugs and diagnostics. Inpatient admissions are $100 \%$ hospital. 
Table 3. Input parameters for estimating PCV vaccine coverage and timeliness

\begin{tabular}{|c|c|c|c|c|}
\hline Parameter & Estimate & \multicolumn{2}{|c|}{ Scenarios } & Source/s \\
\hline $3 \mathrm{~m}$ & $69.9 \%$ & - & - & www.provac-olives.com, Clark and Sanderson Lancet 2009 \\
\hline $9 \mathrm{~m}$ & $91.6 \%$ & - & - & www.provac-olives.com, Clark and Sanderson Lancet 2009 \\
\hline $12 \mathrm{~m}$ & $93.9 \%$ & - & - & www.provac-olives.com, Clark and Sanderson Lancet 2009 \\
\hline $24 \mathrm{~m}$ & $97.8 \%$ & - & - & www.provac-olives.com, Clark and Sanderson Lancet 2009 \\
\hline \multicolumn{5}{|c|}{ Coverage of DTP2 by age in year 2014 (proxy for PCV doses given with DTP2) } \\
\hline $9 m$ & $82.4 \%$ & - & - & www.provac-olives.com, Clark and Sanderson Lancet 2009 \\
\hline $12 \mathrm{~m}$ & $87.0 \%$ & - & - & www.provac-olives.com, Clark and Sanderson Lancet 2009 \\
\hline $24 \mathrm{~m}$ & $94.3 \%$ & - & - & www.provac-olives.com, Clark and Sanderson Lancet 2009 \\
\hline \multicolumn{5}{|c|}{ Coverage of DTP3 by age in year 2014 (proxy for PCV doses given with DTP3) } \\
\hline $3 \mathrm{~m}$ & $0.1 \%$ & - & - & www.provac-olives.com, Clark and Sanderson Lancet 2009 \\
\hline $6 m$ & $41.4 \%$ & - & - & www.provac-olives.com, Clark and Sanderson Lancet 2009 \\
\hline
\end{tabular}

Coverage projections over the period 2014-2023 were estimated by assuming that PCV will achieve the same coverage and timeliness as RV in the early period of introduction, and the same coverage as DTP thereafter. 
Table 4. Input parameters for estimating PCV10 program costs

\begin{tabular}{|c|c|c|c|c|}
\hline \multirow[t]{2}{*}{ Parameter } & \multirow[t]{2}{*}{ Estimate } & \multicolumn{2}{|c|}{ Scenarios } & \multirow[t]{2}{*}{ Source/s } \\
\hline & & Low & High & \\
\hline \multicolumn{5}{|l|}{ Vaccine dose price projection } \\
\hline 2014 & $\$ 0.70$ & - & - & User override \\
\hline 2015 & $\$ 1.40$ & - & - & User override \\
\hline 2016 & $\$ 2.10$ & - & - & User override \\
\hline 2017 & $\$ 2.80$ & - & - & User override \\
\hline 2018 & $\$ 3.50$ & - & - & Model derived \\
\hline 2019 & $\$ 3.50$ & - & - & Model derived \\
\hline 2020 & $\$ 3.50$ & - & - & Model derived \\
\hline 2021 & $\$ 3.50$ & - & - & Model derived \\
\hline 2022 & $\$ 3.50$ & - & - & Model derived \\
\hline 2023 & $\$ 3.50$ & - & - & Model derived \\
\hline \multicolumn{5}{|l|}{ Other vaccine dose costs } \\
\hline International handling (\% of vaccine price) & $0.0 \%$ & - & - & \\
\hline International delivery (\% of vaccine price) & $5.0 \%$ & - & - & Freight costs from GAVI proposal \\
\hline Wastage $(\% \text { of doses discarded, etc })^{\mathrm{a}}$ & $10.0 \%$ & - & - & Estimate - measles vaccine \\
\hline \multicolumn{5}{|l|}{ Safety box cost ( 80 syringes per box) } \\
\hline Price of each safety box & $\$ 0.55$ & - & - & NCDC Geo \\
\hline International handling ( $\%$ of vaccine price) & $0.0 \%$ & - & - & \\
\hline International delivery (\% of vaccine price) & $10.0 \%$ & - & - & Freight costs from GAVI proposal \\
\hline Wastage $(\% \text { of doses discarded, etc })^{\mathrm{a}}$ & $5.0 \%$ & - & - & NCDC Geo \\
\hline \multicolumn{5}{|l|}{ Incremental system costs of introduction ${ }^{\mathrm{b}}$} \\
\hline Incremental system cost per dose & $\$ 0.63$ & - & - & Calculations based on cMYP Georgia 2010-2015 \\
\hline
\end{tabular}

${ }^{a}$ The $\%$ wastage is converted into a factor [1/ (1 - \% wastage)] which is multiplied by the expected number of doses required to meet the anticipated level of coverage.

b Estimated incremental system costs per-dose spending was estimated to be US $\$ 0.63$. The operational launch cost was estimated to be US $\$ 1.06$ in the first year, with costs of US $\$ 0.60$ thereafter 
Table 5. Input parameters for estimating the health impact of PCV10

\begin{tabular}{|c|c|c|c|c|}
\hline \multirow[t]{2}{*}{ Parameter } & \multirow{2}{*}{ Estimate } & \multicolumn{2}{|c|}{ Scenarios } & \multirow{2}{*}{ Source/s } \\
\hline & & Low & High & \\
\hline Dose 1 & $17.0 \%$ & $10.5 \%$ & $34.0 \%$ & Mahon 2006 ratio (Mahon-20\%, Whitney 2006 ratio) \\
\hline Dose 3 & $33.6 \%$ & $20.8 \%$ & $44.3 \%$ & Prymula 2006, Czech \& Slovak Reps. \\
\hline \multicolumn{5}{|c|}{ Vaccine efficacy vs all-cause pneumonia admissions } \\
\hline Dose 1 & $14.4 \%$ & $14.4 \%$ & $20.1 \%$ & Mahon 2006 ratio (Mahon-20\%, Whitney 2006 ratio) \\
\hline Dose 2 & $24.1 \%$ & $24.1 \%$ & $26.2 \%$ & Mahon 2006 ratio (Mahon-20\%, Whitney 2006 ratio) \\
\hline Dose 1 & $41.0 \%$ & $31.9 \%$ & $69.2 \%$ & Mahon 2006 ratio (Mahon-20\%, Whitney 2006 ratio) \\
\hline Dose 2 & $74.5 \%$ & $58.0 \%$ & $90.0 \%$ & Mahon 2006 ratio (Mahon-20\%, Whitney 2006 ratio) \\
\hline Dose 3 & $81.0 \%$ & $63.0 \%$ & $90.0 \%$ & Lucero 2009, Gambia; SA; Philippines; USA (Nat.); USA (Cal.) \\
\hline \multicolumn{5}{|l|}{$\%$ vaccine serotype coverage } \\
\hline Pneumococcal meningitis admissions & $81.0 \%$ & $78.0 \%$ & $82.0 \%$ & Johnson, 2010 \\
\hline Pneumococcal NPNM admissions & $81.0 \%$ & $78.0 \%$ & $82.0 \%$ & Johnson, 2010 \\
\hline Decline in vaccine type coverage $/ \mathrm{yr}^{\mathrm{d}}$ & $5.5 \%$ & $0.0 \%$ & $5.5 \%$ & Assumption \\
\hline
\end{tabular}

${ }^{a}$ Relative coverage is the coverage of those at risk of getting the disease (i.e., effective coverage) compared to coverage of the entire birth cohort (i.e. overall coverage). Overall coverage is multiplied by relative coverage to obtain a more realistic estimate of effective coverage.

${ }^{\mathrm{b}}$ To account for waning duration of clinical vaccine-induced protection, TRIVAC uses a waning matrix with age bands $(<3 \mathrm{~m}, 4-5 \mathrm{~m}, 6-8 \mathrm{~m}, 9-11 \mathrm{~m}, 12-23 \mathrm{~m}, 24-35 \mathrm{~m}$, $36-47 \mathrm{~m}, 48-59 \mathrm{~m}$ ) repeated in the rows and columns of the matrix. The direct protection at the start of each age band is represented by the diagonal from top-left to bottom-right of the matrix. Protection is re-calculated for each age band as the child gets older (moves from left to right in each row). Adjusted protection by age is calculated by adding together the revised protection estimates for each column.

${ }^{\mathrm{C}}$ Rather than endogenous modeling of transmission dynamics, the $\%$ of direct protection for $<5$ yrs is multiplied by a herd effect multiplier (e.g., $120 \%$ ) to give the \% of total protection in the cohort of interest $<5$ yrs. This excludes any herd effect in individuals aged 5 yrs + and is therefore very conservative.

${ }^{d}$ Vaccine type disease replacement is handled by reducing the expected vaccine type coverage in successive vaccinated cohorts by a fixed \% each year, thus reducing overall expected impact of the program in each successive vaccinated cohort by a similar amount. Thus, for a given vaccinated cohort, the \% vaccine type coverage is equal to: $\left[T^{*}(1-R)^{\wedge} \mathrm{N}\right]$ where, $T=\%$ of disease caused by vaccine types in the year of vaccine introduction, $R=\%$ reduction in vaccine type coverage per year following vaccine introduction, and $\mathrm{N}=$ number in the sequence of vaccinated birth cohorts. 
Table 6. Discounted health benefits (10 cohorts vaccinated over the period 2014-2023)

\begin{tabular}{lrrr}
\hline & $\begin{array}{l}\text { No vaccine } \\
\text { (Status quo) }\end{array}$ & \multicolumn{1}{c}{$\begin{array}{c}\text { PCV10 } \\
\text { With vaccine }\end{array}$} & \multicolumn{1}{c}{ Averted } \\
\hline Total outpatient visits & $\mathbf{3 4 , 4 4 4}$ & $\mathbf{2 7 , 2 7 4}$ & $\mathbf{7 , 1 7 0}$ \\
\hline All-cause acute otitis media & 34,444 & 27,274 & 7,170 \\
\hline Total inpatient admissions & $\mathbf{3 4 , 0 4 2}$ & $\mathbf{2 8 , 1 2 2}$ & $\mathbf{5 , 9 1 9}$ \\
\hline All-cause pneumonia admissions & 32,578 & 27,253 & 5,325 \\
Pneumococcal meningitis admissions & 213 & 127 & 87 \\
Pneumococcal NPNM admissions & 1,250 & 742 & 508 \\
\hline Total deaths <5yrs & $\mathbf{1 3 7}$ & $\mathbf{9 6}$ & $\mathbf{4 1}$ \\
\hline All-cause pneumonia admissions & 59 & 50 & 10 \\
Pneumococcal meningitis admissions & 49 & 29 & 20 \\
Pneumococcal NPNM admissions & 29 & 17 & 12 \\
\hline Total children with permanent disability & $\mathbf{4 1}$ & $\mathbf{2 4}$ & $\mathbf{1 7}$ \\
\hline Sequelae group A & 33 & 20 & 14 \\
Sequelae group B & 7 & 4 & 3 \\
\hline DALYs Lost & $\mathbf{4 , 7 4 4}$ & $\mathbf{3 , 3 0 5}$ & $\mathbf{1 , 4 3 8}$ \\
\hline YLDs - DALYS due to morbidity & 666 & 458 & 208 \\
YLLs - DALYs due to mortality & 4,078 & 2,848 & 1,230 \\
\hline
\end{tabular}

Health benefits are discounted at $3 \%$ per year 
Table 7. Discounted economic benefits (10 cohorts vaccinated over the period 2014-2023)

\begin{tabular}{|c|c|c|c|}
\hline & $\begin{array}{l}\text { No vaccine } \\
\text { (Status quo) }\end{array}$ & $\begin{array}{c}\text { PCV10 } \\
\text { With vaccine }\end{array}$ & Averted \\
\hline TOTAL GOV. HEALTH SERVICE COSTS ${ }^{a}$ & $\$ 11,325,355$ & $\$ 9,187,383$ & $\$ 2,137,972$ \\
\hline Total outpatient visit costs & $\$ 407,915$ & $\$ 323,006$ & $\$ 84,909$ \\
\hline All-cause acute otitis media & $\$ 407,915$ & $\$ 323,006$ & $\$ 84,909$ \\
\hline Total inpatient admission costs & $\$ 10,917,440$ & $\$ 8,864,377$ & $\$ 2,053,063$ \\
\hline All-cause pneumonia admissions & $\$ 9,811,714$ & $\$ 8,208,083$ & $\$ 1,603,631$ \\
\hline Pneumococcal meningitis admissions & $\$ 105,341$ & $\$ 62,524$ & $\$ 42,817$ \\
\hline Pneumococcal NPNM admissions & $\$ 1,000,385$ & $\$ 593,770$ & $\$ 406,615$ \\
\hline
\end{tabular}

Costs are discounted at $3 \%$ per year

a Government perspective includes bed days covered by the government and disease-specific diagnostic costs borne by the government at the following health providers: polyclinic, hospital and private individual entrepreneur borne
(PIE). 
Table 8. Discounted cost-effectiveness of PCV (10 cohorts vaccinated over the period 2014-2023)

\begin{tabular}{cr}
\hline & $\begin{array}{l}\text { PCV10 } \\
\text { Government } \\
\text { perspective }\end{array}$ \\
\hline Cost-effectiveness compared to no vaccine & \\
Net cost of vaccine introduction & $\mathbf{\$ 2 , 3 0 0 , 2 4 2}$ \\
Costs of vaccine introduction & $\$ 4,438,214$ \\
$\quad$ Health service costs avoided & $\$ 2,137,972$ \\
DALYs averted & $\mathbf{1 , 4 3 8}$ \\
YLDs averted - DALYS due to morbidity & 208 \\
YLLs averted - DALYS due to mortality & 1,230 \\
US\$ per DALY averted & $\$ 1,599$ \\
Cost-effectiveness threshold & \\
1 x GDP per capita (2012) - WHO threshold for 'highly cost-effective' & $\$ 3,508$ \\
$3 \times$ GDP per capita (2012) - WHO threshold for 'cost-effective' & $\$ 10,525$ \\
\hline
\end{tabular}

Costs and DALYs are discounted at $3 \%$ per year 
Figure 1. US\$ per DALY averted for base case PCV10 scenario and alternative 'what-if' scenarios: Government perspective

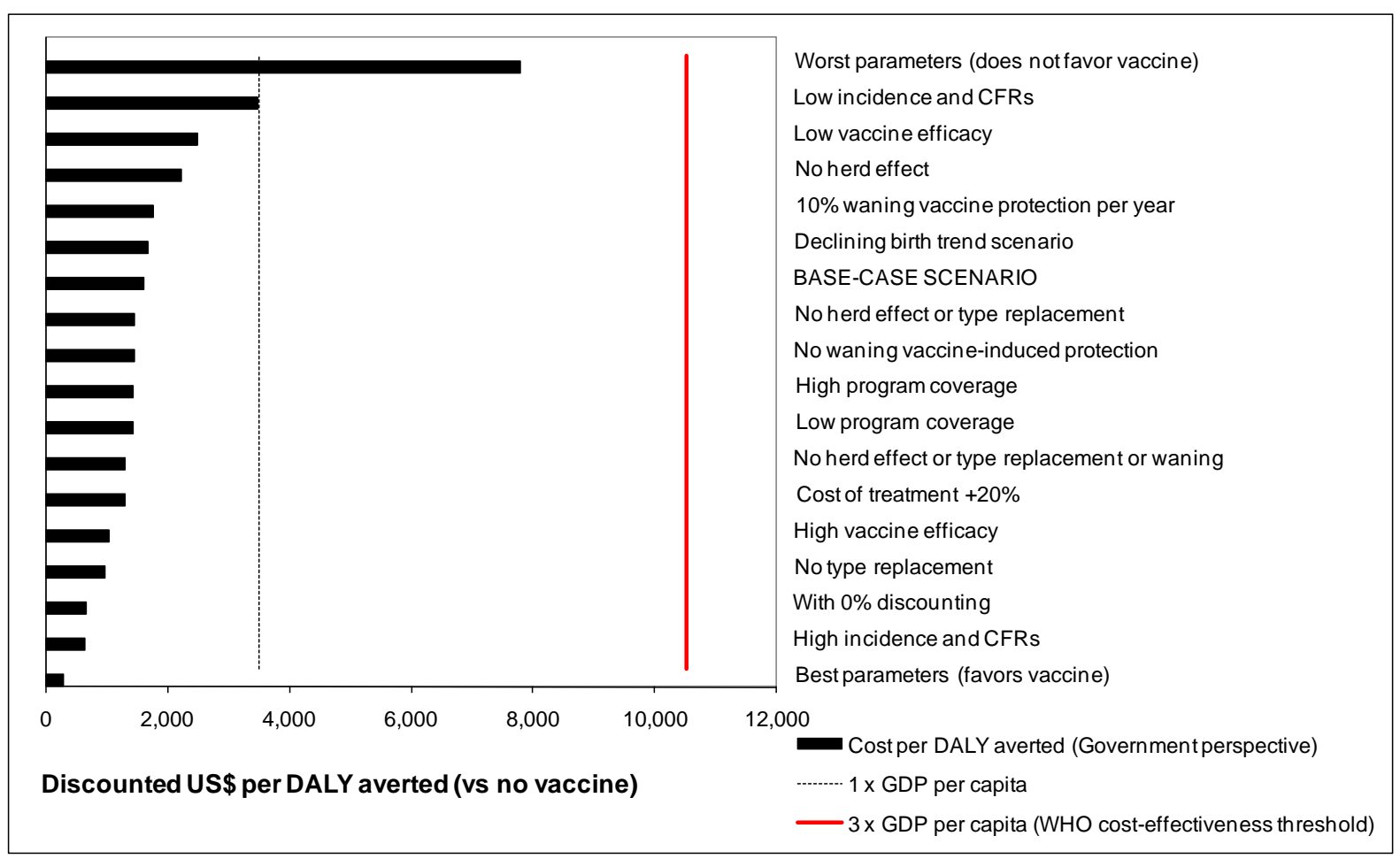

(1) Favorable scenario = High annual incidence rates, high CFRs, low vaccine price projections, low systems costs, high efficacy parameters, $0 \%$ waning in dose efficacy, high serotype coverage parameters, a $125 \%$ herd effect multiplier, a high vaccine coverage projection, high inpatient and outpatient costs.

(2) Unfavorable scenario = Low annual incidence rates and CFRs, high vaccine price projections and system costs, low efficacy parameters, a $10 \%$ yearly waning in dose efficacy, low serotype coverage parameters, low vaccine coverage projections and low outpatient and inpatient costs. 